\title{
I. Ueber die neueren Fortschritte in der chirurgischen Technik.
}

\section{Von Prof. Dr. A. Koehler in Berlin.}

\section{Allgemeiner Theil.}

Die Clin'urgie kann nicht heilen in wissenschaftlichen Simne, sie kaun keine Restitutio ad integrum herbeiführen, die erkı'ankteıl Theile und Gewebe nicht genan so wiederherstellen, wie sie vol' der Erkrankung waren. Das ist lange bekannt und oft her'vol'gehoben; eine Heilung nach der Definition Nothnag'el's (Naturforscherversanmlung 1892) griebt es in der Chirurgie nicht. Wie W. Sachs in einem interessanten Vortrage "Betrachtungen iiber die Grenzeı chirırgischen Könnens" (Basel ınd Leipzig', Sallınanı 1894) sagt, ist es dem Kranken aber auch gleichlgiiltig, ob cr in wissenschaftlichen Simne geheilt ist; wenı es bei lebensgefährlicheı Zuständeı gelingt, durch einen operativen Eingriff die Gefahr zu beseitigen, wenu es gelingt, die dur'ch Verletzingen oder chirur'gische Krankheiten unbrauchbal' gewordenen Theile wieder functionsfähig zn machen, dann ist das eine Heilung in praktischen Sinne. Das Gebiet dieser' Heilungen ist jetzt ein so ausgedehntes und gesichertes, dass die Fortschritte in den Erfolg'en chirurgischen Handelns allgemein anerkannt werden. Diese Erfolge, so gross und so sicher sie heute sind, hängen aber von sehr vielen Bedingungen $a b$; die stärkste Kette hält $1111^{\circ}$ danlı, wenıl alle ihr'e Glieder intact sind.

Einzelne Glieder der Kette, cinzelıe Theile der chir'urgischen Tlätigkeit mögen einmal wichtiger als andere erscheinen; im allgemeinen sind sie gleichwerthig, keines darf ver'nachlässigt wer'den, wenn nicht der ganze Erfolg in Frage gestellt werdell soll. Es ist noch nicht lange her, dass die grossen Fortschritte in der Wundbehandlung die anderen chirnrgischen Anfgaben gering er'scheineı liessen. Wie man operirte, wie man Verbände anlegte, darauf kam weniger all; nuter dem Schutzc der Antiseptik gelang Alles; die peinliche Befolgung technischer und mechanischer Vor'schriften erschien überflüssig, da die Wunden ja doch nuter anti- oder' aseptischen Verbänden heilten. Das war' gewiss ein grosser I'rtlınm; ein schlecht geformiter Amputationsstumpf heilt schlecht trotz aller Aseptik, und eine nicht nach allen Reg'eln der Kunst ansgefïlı'te Arterienuiterbindung gelingt entweder gar niclıt, oder er'st nach ïberfliissigen, vielleicht sehı' schädlichen Nebenverletzung'en.

Dass mall in neuerer Zeit der chirurgischen Technik wieder mehr' Aufmerksamkeit zngewendet liat, gelit schon aus der stattlichen Reihe nener Operationsmethoden und nener Anleitnngen fiil Operationsiibungen hervor. Die ersteren werden wir bei der Besprechung der Operationeı alı einzelıen Körpertheilen erwähıen, von den Lehrbüchern und Anleitungen ${ }^{1}$ ) sei hier nur gesagt, dass sie, was ja in gewisser Beziehnug ein Vorzıg ist fast ansnahmslos rein persönlich sind, d. l. fast nur die eigenen Methoden der Verfasser und höclıstens noch diejenigen einiger' besonder's begünstigter Chirurgen enthalten. Da diese Anleituıgen aus praktischen Grüıdeı nicht zıl umfangr'eich werden dürfen, da sie in ihren Vorschriften möglichst kurz uıd bündig sein müsseı, lässt sich diese Besclı̈änkung nicht vermeiden. Auf breiter anatonischer Basis, voll ganz allgemeinen chirurgischen Gesichtspunkten aus gearbeitet, ist eigentlich nur das Joessel'sche Werk, dessen Weiterführ'ung' und Vollendnng, wie wir hören, in bewäln'ten Händen liegt, und das grosse Leh'buch Farabeuf's. Anl vielseitigsten und fïl' sclinelle Orientirung durch die grosse Zahl guter Abbildungen vorzüglieh geeignet ist die "Techuik“ Esmar'clı's mit ihrer Fortsetzung' (von Esmarch nnd Kowalzig'). - Wir haben lier nur die seit 1990

1) v. Mosetig-Moor'h of, Handbnch der ehirurgischen Technik bei Operationen und Verbänden. Wien 1890. - W. Roser, Chirurgischanatomisches Vademecum, 8. Aufl. Leipzig 1890. - Mac Cormac, Surgical operations. London 1891. - Chauvel, Précis d'opérations de chirurgie. Paris 1891. - Jacobson, The operations of surgery. London 1891. - Rotter, Die typischen Operationen an der Leiche. 2. Aufl. München 1891. - v. Bergmann und Rochs, Anleitende Vorlesung'en fül' den Operationscu'sus an der Ieiche. 2. Aufl. Berliu 1892. Joessel, Lehrbuch der topographisch-anatomisschen Chirurgio unit Eillschluss der Operationsilbungen an der Leiche; I. Extremitäten (1884) II. Brust (1889), III. Banch (1892). Bonn. - Treves, Handbook of sulgical operations (deutsche Uebersetzung von Tenschel. Jena 1892 und 1893). London 1892. - Derselbe, A manual of operative surgery. London 1892. - Kocher, Chirurgische Operationslehre. 2. Aufl. Jena 1894. - Lübker, Chirurgische Operationslehre. 3. Aufl. Wicn 1892. Bell, A manual of the operations of surgery. London 1892. - Krïche Allgemeine Chirurgie und Operationslehre. 5. Aufl. Leipzig 1892. $v$. Esmarch, Handbuch der kriegschirurgischen Technik. 4. Anfl. Von v. Esmarch and Kowalzig neu bearbeitet; dazu der im Jahre 1892 begonnene Ergänzungsband. Kiel 1893. - Clılot, Nouvealıx éléments de chirurgie opératoire. 2. Aufl. Paris 1893. - Farabenf, Précis de manuel opératoire. Paris 1893. - Bayer, Chirurgische Operations. technik. Berlin 1894. - v. Winiwarter, Lchrbuch der chirmlgischen Operationen und der chirurgischen Verbände. Wiesbaden 1894. erschienenen Operationslehren erwähnt und werden auf einzelne, soweit sie nene Vorschläge enthalten, zurückkommen.

Bevor wir nun auf die Fortschritte in der chirurgischen Technik näher eingehen, müssen wir, um Wichtiges nicht zu übergehen, anch die Vorbereitungen zu chirurgischen Maassnahmen und Eingriffen in den Kreis unserer Betrachtungen ziehen. Schon bei der Untersuchung des Kranken, bei der Feststellung der Diagnose, des Sitzes, des Umfanges der Erkrankung sind in den letzten Jahren nicht unwesentliclıe Fortschritte zu verzeichnen gewesen. Auf diesem Gebiete arbeiten die verschiedenen medicinischen Disciplinen gemeinsam; der innere Kliniker, der Psychiater und Neurologe, der praktische Arzt ermöglichen es dem Chirurgen, rechtzeitig einzugreiferl, ehe das Leiden durch Ausdehnung und Complicationen unheilbar goworden ist. Die Vervollkommnung der Untersuchungsmethoden, die Benutzung des elektrischen Lichtes, die Verwerthung physiologischer Thatsachen hat hier, natïrlich in Verbindung mit der verbesserten Wundversorgnng, die Leistungsfähigkeit chirurgischer Eingriffe ganz bedeutend erlı̈ht. Bevor man z. B. Stello und Ausdehnung einer Läsion der Hirnrinde nicht bei intacte in Schädel erkennen konute, bevor man also wusste, wo bei einer bestimmten Reihe von Hirnsymptomen Trepan oder Meissel angesetzt werden mussten, konute von einer zielbewussten operativen Belıandlıng manclıer schweren, ja lebensgefährlichen Erkıankung, wie der Rindenepilepsie, der Hirnabscesse, der Hirntımoren kaum die Rede sein. Wie eifrig auf diesem Gebiete gearbeitet ist, zeigt die grosse Zahl von Veröffentlichungen über die Hirnlokal is ation und ihre Anwendung 1nd Verwerthung in der Chirurgie.') Viele

1) Die nach der 2. Auflage von v. Bergmann's "Chir'urgische Behandlung der Hirnkrankheiten" bis 1891 incl. erschienenen Arbeiten iiber Hirnlokalisation sind von mir im 32. Bande der Deutschen Zeitschrift filr Chirurgie bei Besprechung der Methoden, die Lage und Richtung der Hirnwindungen und Furchen von der Aussenfläche des Kopfes zu bestimmen, genannt. Seitdem liat man sich vielfach mit diesen praktisch wichtigen Untersuchungen beschäftigt; ich kann aber nicht finden, dass die seitdem empfohlenen Methoden leistungsfăhiger und einfacher wären, als die nach der Reid'schen von mir modificirte und zur Construction des "Craniencephalometers" benutzte Methode. - Die Arbeiten sind folgende: Woolonghan, Recherches de la topographie cranio-cérébrale; détermination des rapports du sillon de Rolando et de la scissure de Sylvins avec la boite cranienne. Thèse de Bordeaux 1891. - Vanhersecke, La morphologie des circonvolutions cérébrales (origine, développement, valeur morphologique, physiologique et médical des plis corticaux du cerveau. Thèse de Lille 1891. - Paulier, Détermination de la surface des organes, et du cerveau en particulier, par la méthode des pesées (mensurations faites sul 22 cerveaux). Paris 1892. - d'Antona, Methode, die Centralfurche zil projiciren. Italien. Chir.-Congr. 1892. - Derselbe, Die moderne Chirurgio des Centralnervensystems. Verona 1894. - Krauss, A neurotopographic bust (Gipsbïste mit den Fissuren am Gehirn und den elektromotorischen Punkten an Gesicht und Hals). Journ. of nervous and mental diseases Dec. 1891. - Winkler, Ein Versuch zur Bestimmung der relativen Lageverhältnisse des Grosshirns zur von der Kopfschwarte bedeckten Schädeloberfläche mittels Dreieckconstructionen. Nederl. Tijds. v. Geneesk. 1892 II, 3, Sem. méd. 1892, S. 302 und Zeitschr. f. Heilk., Prag, XIV (Uebersetz. v. Junker v. Lan gegg). - Eine besondere Methode hat Kocher in seiner Operationslehre (s. o.) beschrieben. - In den „Ergeb nissen der Anatomie und Entwickelıngsgeschichte" Bd. 1, S. 91 findet sich eine Besprechung' der craniocerebralen Topographie. - Ferrier's Vorlesungen uber Hirnlokalisation, nbersetzt von Weiss, Wien 1892. Ladame, Note sur une observation de localisation cortico-motrice avec autopsie. Rev. méd. Suisse, Mür'z 1893. - Clado, Topographie craniocérébrale (meist nach Poirier, verwirft den ruban métrique). Sem. méd. 1893, 180, - Warnots, Les fonctions du cerveau. Brisssel 1893. Warnots et Laurent, Les localisations cérébrales et la topographie cranio-encéphalique. Ebenda. -- Cunningham, Beitrag zulr Oberflächenanatomie der Hirnhemisphären mit einem Kapitel über craniocerebrale Topographie nach Hor'slcy, Dublin 1892. - R üdinger, Ueber die Wege und Ziele der Hirnforschung. Mïnchen 1893. - Naecke, Ueber Missbrauch der Lokalisationsthcorie in Psychiatrie und Anthropologie. Neurol. Centralbl. 1. Oct. 1893. -- M. E. Masse, Sur la topographie cranioencéphalique. XI. intern. med. Congress. - Adamkiewicz, Tafeln zur Orientirung an der Gehirnoberfläche des lebenden Menschen bei chirurgischen Operationen und klinischen Vorlesungen. Wien 1894, 2. Aufl. Harda, An improved method of brain localisation in epilepsy (Reizung mit dem elektrischen Strom). Ann. of surg., Februar 1894. - Hirth, Die Lokalisationstheorie angewandt auf psychologische Probleme. München 1894. - Lannelongue et Manclaire, Neuc praktische Methode der cranio-cerebralen Topographie bei Kindern. Sem. méd. 1894, S. 460 - v. Beck, Beiträge zur Pathologie und Chirurgie des Gehirns. Tutbingen 1894. - Delvoie. Histoire, indications et contre-indications, teclimique et résultats de la trépanation cranienne. Briissel 1893. - Wir haben bei dieser Zusammenstellung alle casuistischen Mittheilungen iiber Fälle von Verletzungen, Abscesse und Tumoren des Gehirns, bei denen diese oder jene Methode der Iokalisation gebraucht war, sowie die Mittlei- 
von den mühsam erprobten und empfohlenen Methoden sind zu complicirt, um allgemeine Verbreitung zu finden, und dabei doch nicht sicherer als andere, welche einfach und leicht zu benutzen sind. Abweichungen von 1 oder $1^{1 / 2} \mathrm{~cm}$ werden, oft schon durch individuelle Verschiedenheiten bedingt, leicht vorkommen, haben aber auch keinen besonderen Nachtheil, da die Oeffnung im Schädeldach, mag man Trepan oder Meissel benutzen, wohl immer einen grösseren Durchmesser haben wird. Ganz besonder's gilt dies bei Benutzung der sog. temporären Resections-Methoden. In der v. Barde. leben'schen Klinik hat sich der nach der Reid'schen Methode construirte Craniencephalometer sowohl zu Demonstrationen, als auch bei Trepanationen wegen verschiedener Hirnkrankheiten sehr gut bewährt (s. u.)

An der chirurgischen Behandlung der Hirnkrankheiten lässt sich der durch Verbesserung der Untersuchungsmethoden bewirkte Fortschritt am besten demonstriren; vorhanden ist er natürlich bei allen Krankheiten und am bekanntesten wohl in der Bauchchirurgie. Frühzeitige Diagnosen ermöglichen hier oft einfachere Operationsmethoden und bessere Erfolge, wo sonst, bei späterem Operiren, die Prognose sehr traurig war. Es ist bekannt, dass in der Bauchchirurgie Anti- und Aseptik nicht jene fast absolnte Sicherheit gewähren, auf welche wir im allgemeinen zu rechnen gewohnt sind. Ein grosser Theil dieser Misserfolge wird beobachtet, wenn sich schon Zustände herausgebildet haben, gegen welche unsere Kunst nichts ansrichten kann; sei es die wegen eingetretener Complicationen zu sehr in die Länge gezogene Operation, oder seien es infectiöse, durch die Laparotomie nicht mehr zu beseitigende Zustände. In beiden Fällen wird nan oft die Ueberzeugung gewinnen, dass eill früherer Eingriff bessere Aussichten gehabt hätte. Wir erinnern an die Stenosen des Pylorus, an die Perityphlitis (jetzt Appendicitis), an Ileus, Wanderniere, Bauchtumoren, an die Geschwülste der Harnblase, die Erkrankungen des Pankreas - - auf diesem gemeinsamen Arbeitsfelde des inneren und des chirurgischen Arztes ist in den letzten Jahren durch Vervollkommnung der Untersuchungsmethoden und dadurch möglich gewordenes frühzeitiges Operiren viel erreicht. Vorläufig können wir allerdings in manchen Fällen die Probelaparotomie, ein nur scheinbar heroisches Untersuchungsmittel, nicht entbehren; sie allein ist oft imstande, die Diagnose zu klären und damit das weitere Vorgehen zu bestimmen. Die Probepunctionen sind, wenn die eigentliche Operation nicht sofort folgen kann, gefährlich, lassen zuweilen im Stich oder führen zu Irrthümern, weil man nicht immer mit Bestimmtheit sagen kann, dass das Entleerte wirklich aus der zu untersuchenden Partie stammt. Die Probeincision ist wohl ein grösserer operativer Eingriff, dabei aber ungefährlich und viel leistungsfähiger als die Punction; sie bildet, wenn die Operation folgen soll, schon einen Theil derselben, und wenn sich die Unmöglichkeit oder Aussichtslosigkeit der Operation herausstellt, dann bringt die Probeincision keinen Nachtheil, da die Wunde sich durch erste Vereinigung schliesst. Wir wollen übrigens nicht unerwähnt lassen, dass in manchen Fällen, in denen nichts oder nichts "Operables" gefunden war, auch ohne dass Hysterie vorhanden gewesen wäre, nach der einfachen Probeincision ganz bedeutende Besserung, ja Schwinden der vorher grossen Beschwerden beobachtet ist. Man nennt das: die Heilwirkung einer Operation an sich; eine Erklärung giebt es nicht dafür. (Vergl. die Dissertation von 0 . Küttner, Berlin 1893, Ueber die Erfolge operativer Eingriffe an sich, und Maylard, Glasgow med. Journ. October 1891).

Die sonstige Vorbereitung des Kranken, des chirurgischen Personals und Materials, die Wundversorgung im allgemeinen hat seit der Modification der Antiseptik zur Aseptik, d. h. seitdem man $v$ or der Operation streng antiseptisch und während derselben nur aseptisch verfahrt, nicht mehr mit antiseptischen Lösungen, am besten überhaupt nicht spült, keine wesentlichen Aenderungen erfahren. Die Zusammenstellung der darauf sich beziehenden Arbeiten ist von mir seit einer Reihe von Jahren in der Einleitung zu den Berichten über die v. Bardeleben'sche Klinik (Charité-Annalen, Jahrgang 188̄́-1893) als „Chronik der Wundbehandlung" gegeben. Ausführlicher sind dieselben von mir in der Deutschen Militärärztlichen Zeitschrift, 1893 (Neuere Arbeiten über Wundbehandlung) besprochen.

In derselben Zeitschrift findet sich auch eine Besprechung der neueren Arbeiten über Anaesthetica, bis zum Jahre 1892 incl. Seit dieser Zeit sind wieder unendlich viele experimentelle und klinische Mittheilungen über die Narkose veröffentlicht; ein Beweis, dass die Meinungen darüber noch weit auseinandergehen. Nach den Ergebnissen der grossen Sammelforschung der deutschen Gesellschaft für Chirurgie schien es, als ob der Aether dem Chloroform bei weitem lungen über die Centren einzelner bestimmter Functionen nicht erwuhnt, sondern nur die allgemeinen Arbeiten tiber diesen Zweig der Hirnchirurgie aufgezählt. vorzuziehen sei; die ZahI der Narkosen mit Aether ist aber immer noch so viel geringer, als die Zahl derjenigen mit Chloroform, dass wenige ungünstige Erfahrungen mit Aether das Verhältniss ändern können. Sehr Jehrreich sind in dieser Beziehung die Mittheilungen Poppert's in dieser Zeitschrift (13. September 1894) ïber einen F'all von Aethertod infolge von Lungenödem nebst Bemerkungen zur Narkotisirungsstatistik. Auch Herhold berichtet über einen Todesfall in der Aethernarkose (Berliner klin. Wochenschrift, 2. April 1894). ${ }^{1}$ ) Dieselbe Frage behandelt Selbach, Bonn 1894: Ist nach länger dauernden Aetherinhalationen eine tödtliche Nachwirkung derselben zu befürchten? - Ueber die Einwirkung des Aethers auf die Nieren finden wir Bemerkungen bei Garrè (Münch. med. Wochenschr. 1891, No. 7, 65. Naturforscherversanmlung, Bruns Beitr. Bd. XI); nach ihm ist dabei transitorische Albuminurie nicht häufiger, als bein Chloroform; bei $\mathrm{Ba}$ rensfeld (Münch. med. Wochenschr., 9. October 1894). Ueber das Vorkominen von Eiweiss im Urin nach der Athernarkose bei Fuster, welcher in der Klinik Dumont's die Narkose leitete, in der deutschen Zeitschr. f. Chir. Bd. 29; er leugnet das Vorkommen der Albuminurie nach der Aethernarkose. D n mont ist ein eifriger Vertheidiger des Aethers (Correspondenzbl. für Schweizer Aerzte 1883, No. 23 nnd Festschrift für Kocher, Wiesbaden 1891); er benutzt eine Modification der Julliar d'schen Maske (Monatsschr. f.̈̈rztl. Polyt. April 1887). Wenn auch die Zahl der den Aether empfehlenden Chirurgen eine ganz bedeutende ist, so fehlt es doch nicht an solchen, welche davor warnen, das Chloroform zu verlassen. Schon Garrè (s. o.) sagt: "Nicht Aether od er Chloroform, sondern Aether und Chloroform"; "Clarke, New-York med. Rec. 14. April 1894 ist gegen den Aether, ebenso v. d. Goltz, New-Yorker ined. Presse, März 1888 und Kappeler, 19. Congr. d. deutschen Gesellsch. für Chir. 1890. - Aus alledem geht die Nothwendigkeit hervor, die grossen Sammelforschungen ïber Narkosen fortzusetzen, welche bisher wenigstens das ergeben haben, dass es nicht angängig ist, ein Anästheticum für alle Fälle brauchen zll wollen: für Kinder, für Hals- und Lungenkranke, für Operationen am Gehirn, an Nase, Mund und Hals (Kropf!), für Operationen bei künstlicher Beleuchtung eignet sich der Aether nicht; bei Herzkranken ist er dem Chloroform vorzuziehen. $\mathrm{Ob}$ er wirklich im allgemeinen ungefährlicher ist, als dieses, ist noch nicht mit genügender Sicherheit festgestellt. - Dass Pental und Bromäthyl keinem Anästheticum an Gefahr nachstehen, ist schon jetzt als sicher zu betrachten. - Wenn man Kinder ind aufgeregte, ängstliche Erwachsene aussehliesst, dann kann für eine Reihe von Operationen auch die örtliche Anästhesie benutzt werden. Eine Art derselben, die von Schleich empfohlene Infiltrationsanästhesie (Schmerzlose Operationen, Berlin, Springer 1894, Berl. klin. Wochenschr. 1891, No. 35 und Therap. Monatshefte, September 1894) erfuhr nur wegen der eigenthümlichen Form, in welcher diese Empfehlung geschah, auf dem 21. Congress der Deutschen Gesellschaft für Chirurgie eine scharfe Abweisung. Auch für diese Methode wird immer nur ein sehr beschränkter Wirkungskreis vorhanden sein. (Vergl, die oben erwähnte Zusammenstellung über neuere Anästhetica in der Deutschen mil.-ärztl. Zeitschrift). Ueber Operationen ohne Anästhetica berichtet Keetley in der Royal Soc. vom 14. November 1893.

Eine in letzter Zeit wieder mehr betonte Schädlichkeit der allgemeinen Betäubung, welche übrigens mit dem Betäubungsmittel nichts zu thun hat, ist die Lähmung nach der Narkose, eine Drucklähmung bei zu weit nach hinten und oben gehaltenem Arm. B üdinger, Langenbeck's Archiv Bd. 47, Braun, diese Wochenschrift 1894, No. 3 und Gaupp, Centralbl. f. Chir. 1894, No. 34 haben diesen Gegenstand bearbeitet. Es genügt wohl, die Gefahr und ihre Ursache zu kennen, um sie sicher zu vermeiden.

Ueber Methoden und Apparate zur Narkose wollen wir hier nur einiges hervorheben. Laborde und Guérin hatten die alte Theorie wieder besprochen, nach welcher die primären Zufälle bei der Chloroformbetäubung reflectorisch durch Reizung der Trigeminusendigungen in der Nasenschleimhaut hervorgerufen würden (Sem. méd. 1893, S. 339). Casas ovici (ebenda, S. 214) sucht diese Reizung durch Cocainisirung der Nasenschleimhaut vor der Narkose zu vermeiden. Denselben Vorschlag machte Rosenberg in der Berliner medicinischen Gesellschaft am 14. November 1894. Ob man dadurch die Synkope verhütet, müssen weitere Erfahrungen lehren. Latta (Sem. méd. 1894, S. 212) empfiehlt bei Gebärenden eine Mischung von 90 Theilen Chloroform mit 1 Theil Amylnitrit; Langlois und Maurange (ebenda, S. 321) und Diousidon

1) Aus früherer Zeit verweisen wir auf: Brit. med. Journ. 1886 I, S. 556 und Med. News 19. November 1887 (je ein Fall); Jakob berichtete im Brit. med. Journ. 1886 I, S. 489 uber zwölf Chloroform- und drei Aethertodesfälle; Agnew in Philad. med. News 1887, No. 21 uber einen Todesfall in der Aethernarkose; ebenso $\mathrm{Mc} \mathrm{K}$ ine in New-York med. News 1. Juni 1889; ein| weiterer Fall findet sich in der Lancet 1889 I, S. 800. 
(Thèse de Paris 1894) empfehlen eine Injection von Sparteinum sulfuricum 0,03 und Morphium 0,01 eine Viertelstunde vor der Narkose. Cathoile (Thèse de Lyon 1894) besclıreibt dagegen die Gefahren der gemiscliten Narkose, vor welcher aucli Garrè (l. c.) u. a. warnen. - Neue Apparate empfehlen IV. Vajula (Pest. med. Presse 1894, No. 4) Narkotisirungsapparat aus Glas; Dubo is (Sem. méd. 1893, S. 390); Rosenfeld (Wien. med. Wochenselı: 1893, No. 28) ein neuer Narkosenkorb (für Aether); Rosenberg (Deut. med. Woclienschr. 1894, No. 29) eine neue Chloroformmaske, an welcher die Tropfflasche befestigt ist. Diese combinirten Apparate, welche schon mehrfach empfohlen wurden, werdell bei lieftig sich sträubenden Patienten, besonders im Excitationsstadium, schwer zu gebrauchen sein. In No. 48 des Centralblattes fül Chirurgie 1894 empfiehlt RitschI für die Aetlernarkose eine Maske aus Metall mit Glasfenstern.

Den Schluss dieser „vorbereitenden Operationen" bildet die Verhütung des Blutverlustes. Bei einer ganzen Reihe von Amputationen und Exarticulationen kann man nach Art der RoseLü cke'schen Methode der Oberschenkelexarticulation den Blutverlust auf eill sehr geringes Maass beschränken, wenll man an der Stelle, an welcher das Glied abgesetzt werden soll, beim $\mathrm{Be}-$ ginn der Operation die Hauptarterie und Vene doppelt unterbindet und $z$ wisclien den Ligaturen durchschneidet. Bei einzelnen Operationen, z. B. bei der Exarticulatio humeri, genu, bei der Grittischell Amputation, kann man so verfalıren, dass die Durchschneidung der grossen Gefässe den letzten Act bildet. Ein Assistent lält dabei den die Gefässe enthaltenden Lappen mit beiden Händen fest und kam, wie wir sehr häufig festgestellt haben, die Gefässe dabei so gut comprimiren, dass kein Tropfen Blut verloren gelit und die Isolirung und Unterbindung vor dell comprimirenden Fingern in aller Rulle vorgenommen werden kamn. Das sind einige Ersatzmittel der Esmarch'schen Blutleere füı Fälle, in denen kein elastisches Umschnürungsmaterial vorhanden ist, oder in denen man aus irgend einem Grunde darauf verzichtet. Dass man die Umschnürung auch nit leinenen Binden ausfülıren kaun, welche am besten (nacl Elevation) trocken angelegt und dann von der Peripherie her befeuchtet werden, ist von v. Bardeleben und R. Köhler vor langer Zeit bewiesen und seitdem oft il der Klinik demonstrirt. Neuber empfall dasselbe Verfahren auf dem Congresse der Deutschen Gesellschaft fül Chirurgie im Jalıre 1893 (s. Langenb. Archiv Bd. 46: Asepsis und künstliche Blutleere). Während v. Esmarch auf die elastische Unischnürung des ganzen Gliedes nur in wenigen Ausnahmefällen verzichten will, neigt eine grosse Zahl der Chirurgen dazu, sich auf Elevation und Umschnïrung mit dem Schlauch oder dem (Nicaise'schen) Gummibande zu beschränken (velgl. den Briefwechel zwischen v. Esmarch und Senn in New York med. Record rom 20. Mai 1893: Some further remarks on elastic constriction as a haemostatic measure, with a letter from Prof. v. Esmarch, und EggeI, Dissert. Berlin 1891: Ueber die Ersatzmittel der' Esmarch'schen küisstichen Blutleere). - Mc Leod empfiehlt nach Art der Wyethschen Methode der Blutleere bei Exarticulatio femoris und nach Art der bekanntell Abschnürung grösserer Geschwülste durch eine elastische Ligatur, welche hinter mehreren, all der Basis durchgestossenen Nadeln angelegt wird, die Acupressur, die temporäre percutane Umstechung, oder die Acupressur in Verbindung mit elastischer Umschnulrung (Lancet, 19. April 1890: The skewer' method of preventing haellorrlage during operations). - Eine neue "hämostatische Pincette", bei welcher die scliwer zu reinigenden Rinnen am Fassende beseitigt sind, empfiehlt Gil'ard (Monatsschrift fül ärztl. Polyt., Mai 1887). - Dass man Blutungen naclı Geschwulstexstirpationen, bei denen das Fassen einzelner Gefässe schwer möglich ist, durch exacte Jodoformgazetamponade stillt; dass man bei seitlichen Verletzungen grosser Gefässe die Naht des Schlitzes, die seitliche Unterbindung und in Fällen, wo beides nicht möglich ist, das Fassen des Schlitzes mit Liegenlassen der Pincetten in einer grossen Reile voll Fällen mit bestem Erfolge ausgeführt hat, wollen wir nur kurz erwähnen. Eine Errungenschaft der neueren Zeit ist es auch, dass man die Verletzungen der Vena femoralis in der Leistenbeuge entweder durch die genannten Verfahren, oder, wo es nicht angängig ist, durch Resection des verletzten Abschnittes (Rose's blutdichte Exstirpation auf die Venen angewandt) zur Heilung bringt. Man kann wohl als sicher annehmen, dass der Verschluss dieser Vene keine bösen Folgen liat, wenn es nur gelingt, die Wunde aseptisch zu halten, d. h., wemn nicht durch entzündliche Schwellung in und an der Wunde die Bildung collateraler Balınen zu sehr erschwert wird. (Vergl. die oben genannten Berichte über die v. Bardeleben'sche Klinik und die betreffenden Abschnitte im Jahresberichte von VirchowHirsch.) Es ist wohl nicht überflüssig, auf diese Thatsachen noch einmal hinzuweisen, weil immer wieder mühsane Arbeiten unternommen werden, um die angeblich unvermeidlichen bösen Folgen dieser Verletzung wissensclaftlich zu erklären, obgleich die klinische Erfalırung darüber längst Klarheit geschaffen und bewiesen laat, dass man sogar grosse Stücke aus der Vena femoralis reseciren kann, olıne dadurch Gangrän des Beines zu bewirken.

Die Veränderungen, welche in letzter 'Zeit an den täglich gobrauchten Instrumenten vorgenommen sind, beziehen sich fast alle darauf, dieselben der aseptischen Wundbehandlung anzupassen. d. h. so einzurichten, dass sie bequem der Wirkung des kochendell Wassers, resp. der Sodalösullg' ausgesetzt werden können. Die alten Instrumente mit hölzernen Stielen sind dazu natürlich nicht zu gebrauchen. Bei Scheerell, Zangen und älnnliclien nicht ganz einfachell Werkzeugen ist man bestrebt, sie zerlegbar zu machen, weil sie sonst trotz aller Sorgfalt an einzelnen Stellen rosten und weniger brauchbar werdell. Wenn es auch ein Irrtlum ist, dass eine Scheere nur "kneift" und nicht schneidet, und wem man auch dieses Schneiden bei der Scheere durch ein sanftes Zurückziehen beim Zusammendrücken lloch glatter machen kaml, wie v. Bardeleben im I. Bande seines Lehrbuchs (8. Ausgabe) lervorhebt, so ist es doclı als ein Fortscluritt zu bezeiclnen, dass man jetzt Scheeren lielstellt, deren Arme sicl beim Schneiden an einander verschieben. Am bekanntesten ist die von der Firma Henkels fabricirte Form, welche nur den Nachtheil hat, theuer und schwer zu reinigen zu sein. Wir haben nach dem Prinzip der sogenannten amerikanisclıen Rosenscheere Verband- und andere Scheeren von Détert anfertigen lassen, welcle sich seit mehreren Jahren recht gut bewährt haben; beim Senleifen muss jeder Scheerenarm bis hinter das Scliloss gleichmässig abgeschliffen werden, sonst liegen die Blätter einander nicht an (s. Verhandlungen der freien Vereinigung der Chirurgen Berlins vom 16. November 1892). - Natürlich werden auch Pincetteu, Kornzangen, Spritzen und viele andere Instrumente so gemacht, dass sie auseinanderzunelımen und bequenı zu reinigen sind; so lange nicht die nothwendige Festigkeit und Haltbarkeit darunter leidet, sind alle diese Instrumente sehr brauchbar. Dass aber die schweren metallenen Behälter, in welche die früllere Verbandtasche verwandelt ist, ersetzt werden durch Taschen aus leichterem sterilisirbarem Stoff, wie es auch schon empfohlen ist, scheint mir nur cine Frage der Zeit zu sein. Für silberne Katheter, welche bekanntlich an besten aus einem Stück bestehen, dann aber nicht gut zu transportiren sind, liaben wir bei Engmann (Charitéstrasse) handschuhähnliche Behälter herstellell lassen, welche aus starkem Drillich mit Asbesthröhren bestehend, leicht und sicher zu sterilisiren sind. In ihnen werden die Katheter aufbewahr't und transportirt.

Von den sogenannten El em en tar-Operationen kommt hauptsächlich die Incision und die Wundnaht für uns in Betracht, da sich bei den anderen, der Punction, der Ligatur, der Cauterisation u. s. w. nur insoferlı manches geändert lat, als ihr Wirkungskreis allmählich geringer geworden ist. - Fül die Incisionen wird mit Recht eine bestimmte Richtung enpfollen; besonders Kocher giebt darüber in seiner Operationslehre (s. o.) ausführliche Vorschriften. All selır vielen Kör'per'stellell entspricht die Spannung. der Hant dem Verlaufe der Nerven und Gefässe, so dass die Lage des Hautschnitts an diesen Stellen ganz bestimmt vorgeschrieben ist. Die Befolgung der betreffenden Vorschriften, welche Koche $\mathbf{l}^{\circ}$ noch durch mehrere klare Abbildungen erläutert, sichert nicht nur eine glattere Heilung, sondern auch eine bessere Narbenbildung. Mit Recht wird darauf hingewiesen, dass man z. B. am Halse (oberer und unterer "Kragenschnitt", Querschnitt im Trigonum supraclaviculare) die Narben richtig angelegter Incisionen später kaum zu finden vermag, während sie in anderen Fällen fast keloidartig. werden und direkt verunstaltend wirken. Damit ist natürlich nicht gesagt, dass Stelle und Richtung des Hautschnittes das allein Maassgebende für die spätere Narbenbildung sei. Auch für die Frage der Bauchbrüclıe nach Operationen am Unterleib kaun die Schnittrichtung von Bedeutung sein, obgleich bei ihnen wohl auch die Art des Nähens selır in Betracht kommt. Ein sicleres Urtheil lässt sich darüber noch nicht abgeben; man weiss, dass es zuweilen selır lange dauert, bis die Hernie sich bildet. - Der Hautsclunitt ist oft der erste Act einer längeren Operation, und voll dem Zwecke der letzteren hängt die Grösse der Oeffinung in der Haut ab; von den möglichst kleinen Schnitten ist man neuerdings ganz abgegangen: was vernälıt werden und durch erste Vereinigung heilen kamn, das kann auch ausgiebig gespalten werden; dann wird man am besten in der Tiefe arbeiten und unnötlige Nebenverletzungen vermeiden kömnen. - Für die Wuıdnalıt sind sowohl verschiedene neue Instrumente, Nadelhalter und NadeIn, als auch mehrere Vorschläge für die Art des Nähens zu verzeichıen; ausserdem hat man mehrfach versucht, eine Vereinigung ohne Nalıt herbeizuführen. Credé's Vor'schläge auf dem 23. Chirulgen-Congress, die Naht bei Amputationsstümpfen durch die Art des Verbandes zu ersetzen, Berr'u t's Appareil unitif (VII. franz. Chir. Congr.), welcher' zu ähnlichenı Zwecke empfohlen wurde, und Lignac's Methode 
(Sem. méd. 1893, S. 80) mit Serres fines und Collodium (sobald das Collodium erstarrt ist, sollen die Serres entfernt werden) das alles ist natürlich nur an Stellen möglich, wo gar keine Spannung vorhandell ist. Diese unblutigen Vereinigungsmittel haben bekanntlich ihre zahlreichen Vorgänger, besonders aus der sogenannten vorantiseptischen Zeit, als man den „Fremdkörper im Nahtcanal“ noch mit Recht fürchtete. Unangenehme Erfahrungen sind hier und da auch in unserer Zeit bei der Wundnaht gemacht worden; daher die fast unzähligen Versuche über die Sterilisirung der zahlreichen Arten von Nahtmaterial. Kocher hat das Catgut ganz verlassen; viele andere sind ihm gefolgt. Halsted (Johns Hopkins Hospital-Rep. II, No. 5) und $\mathrm{P}_{0} \mathrm{z}$ z i (Sem. méd. 1892, S. 72) glauben, dass die Sticheanaleiterungen mit ihren Folgen zu vermeiden sind, wenn man bei der Naht die Haut selbst nicht mit durchsticht, sondern ,intracutan" näht. Alles Vorschäge, welche erst recht dazu auffordern müssen, die Methoden der Sterilisirung des Materials und der antiseptischen Reinigung der Operationsstelle und aller Dinge, welche mit der Wunde und ihrer Umgebung in Berührung kommen, weiter zu vervollkommnen. - Um das Einkrämpen der Wundränder bei der Naht zu verhindern und um diese selbst zu erleichtern, empfiehlt Guinard (Bullet. de la Soc. de chir., 1. Februar 1893) eine Pincette mit breiten Fassenden. - Die neu empfohlenen Nadelhalter sind durchweg Modificationen älterer Modelle; so hat Haged orn selbst seinen sehr brauchbaren Halter für platte Nadeln dahin verbessert, dass er leichter auseinanderzunehmen und zu reinigen und dabei doch stärker geworden ist (Centralblatt für Chirurgie 1890, No. 10); B u rrage empfiehlt (Bost. med. Journ., 18. September 1890) eine Modification des Sims'schen, Adams (Glasgow. med. Journ., April 1889) eine solche des Haged orn'schen Halters; Hüpeden (Berliner klin. Wochenschrift 1893, No. 30) empfiehlt als ,aseptisches Nadelzeug“ eine Art Magazinnadel, welche leicht zu sterilisiren ist; bei dieser, wie bei dem von $\mathrm{Kurz}$ (Centralblatt für Chirurgie 1893, No. 13) als Nadelpistol empfohlenen Instrument, bei welchem die Nadel rechtwinkelig zum Stiel gestellt wird, befindet sich das Oehr in der Nähe der Spitze. Die Nadel von De Moij (Nederl. Tijdschr. 1890, No. 10) hat statt des Oehres einen seitlichen Spalt in der Nähe der Spitze; der Faden wird nach Durchführung der Nadel durch die Wundränder in den Spalt eingehängt und die Schlinge durch den Nahtcanal zurückgezogen. Dadurch, dass der eine der beiden freien Fäden durch die Schlinge geführt und mit dem anderen verknotet wird, soll ein besonders sicherer Verschluss der Wunde von der Oberfläche zur Tiefe gewährleistet sein. Hohlnadeln, nach Art der in Vergessenheit gerathenen Simon'schen - aber ohne Stiel — sind von mir (Hohle Nähnadeln für Draht und Setole, Centralblatt für Chirurgie 1893, No. 27) und von Créquy (Bull. de therap., 15. December 1889, als Probetroicart) empfohlen.

Wer sich eingehend über die Art der Näh te (Knopfnaht, fortlaufende Naht, Matratzennaht u. s. w.) orientiren will, dem ist Esmarch's Technik (s. o.) und der Artikel von Farguhar Curtis im New-York med. Rec. vom 26. October 1889 zu empfehlen. Auf der v. Bardeleben'schen Klinik ist seit einer Reihe von Jahren die sogenannte Languettennaht, eine Art fortlaufender Naht, bei welcher die Wundränder nicht, wie bei der gewöhnlichen „überwendlichen" Naht schräg, sondern in querer Richtung von einander gezogen werden, in Gebrauch. Eine gute Zusammenstellung der verschiedenen Arten der secundären Naht giebt Metzlar (Nederl. Tijdschr., 1. August 1891). - Ueberall, wo sonst Etagennähte angelegt wurden, haben wir mit bestem Erfolge die verschiedenen Schichten von der Tiefe zur Oberfläche mit einem einzigen langen Catgutfaden und fortlaufender Naht - die Haut wieder mit der Languettennaht - vereinigt. Besonders leistungsfahig hat sich uns diese Art der Etagennaht bei der Vereinigung der Bauchwunde nach der Laparotomie gezeigt.

Ueber die grosse Zahl verschiedener Substanzen, welche als Naht- und Unterbindungsfaden zubereitet werden, giebt unsere oben erwähnte "Chronik der Wundbehandlung" eingehende Auskunft. 